\title{
The Complex Morphology of the Young Disk MWC 758: Spirals and Dust Clumps around a Large Cavity
}

\author{
Y. Boehler ${ }^{1}$ (D) L. Ricci ${ }^{1}$, E. Weaver ${ }^{1}$, A. Isella ${ }^{1}$ (D) M. Benisty ${ }^{2}$ (D) J. Carpenter ${ }^{3}$ (1) C. Grady ${ }^{4}$, Bo-Ting Shen ${ }^{5}$ (D),

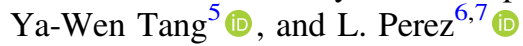 \\ ${ }^{1}$ Rice University, Department of Physics and Astronomy, Main Street, 77005 Houston, USA \\ ${ }^{2}$ Universite Grenoble Alpes, CNRS, IPAG, F-38000, Grenoble, France \\ ${ }^{3}$ Joint ALMA Observatory (JAO), Alonso de Cordova 3107 Vitacura-Santiago de Chile, Chile \\ ${ }^{4}$ Exoplanets and Stellar Astrophysics Laboratory, NASA Goddard Space Flight Center, Greenbelt, MD, USA \\ 5 Academia Sinica, Institute of Astronomy and Astrophysics, Taipei, Taiwan \\ ${ }^{6}$ Max-Planck-Institute for Astronomy, Bonn, Germany \\ ${ }^{7}$ Universidad de Chile, Departamento de Astronoma, Camino El Observatorio 1515, Las Condes, Santiago, Chile \\ Received 2017 October 20; revised 2017 December 11; accepted 2017 December 11; published 2018 February 1
}

\begin{abstract}
We present Atacama Large Millimeter Array observations at an angular resolution of 0 !" $1-0$ ".2 of the disk surrounding the young Herbig Ae star MWC 758. The data consist of images of the dust continuum emission recorded at 0.88 millimeter, as well as images of the ${ }^{13} \mathrm{CO}$ and $\mathrm{C}^{18} \mathrm{O} J=3-2$ emission lines. The dust continuum emission is characterized by a large cavity of roughly 40 au in radius which might contain a mildly inner warped disk. The outer disk features two bright emission clumps at radii of $\sim 47$ and 82 au that present azimuthal extensions and form a double-ring structure. The comparison with radiative transfer models indicates that these two maxima of emission correspond to local increases in the dust surface density of about a factor 2.5 and 6.5 for the south and north clumps, respectively. The optically thick ${ }^{13} \mathrm{CO}$ peak emission, which traces the temperature, and the dust continuum emission, which probes the disk midplane, additionally reveal two spirals previously detected in near-IR at the disk surface. The spirals seen in the dust continuum emission present, however, a slight shift of a few au toward larger radii and one of the spirals crosses the south dust clump. Finally, we present different scenarios to explain the complex structure of the disk.
\end{abstract}

Key words: infrared: planetary systems - ISM: individual objects (MWC 758) - planet-disk interactions protoplanetary disks - stars: pre-main sequence - submillimeter: planetary systems

\section{Introduction}

Planets form from the gas and solids contained in disks surrounding young stars. Once enough mass is assembled into a protoplanet, the gravitational interaction between this object and material in the disk is expected to generate a variety of substructures in the disk itself, as rings, spirals, or cavities (Kley \& Nelson 2012; Baruteau et al. 2014; Dipierro et al. 2016). The formation of these substructures might in turn dramatically affect the formation of other planets through the creation of gas pressure maxima able to concentrate dust particles (Pinilla et al. 2015; van der Marel et al. 2015; Guilera and Sándor 2017).

Observations of disks in nearby star-forming regions at millimeter wavelengths and at high angular resolution have the capabilities to reveal these substructures across all the disk vertical height. The Atacama Large Millimeter Array (ALMA) is currently the most suited telescope for such observations and has unveiled azimuthal asymmetries (Pérez et al. 2014; van der Marel et al. 2013; Boehler et al. 2017), rings (ALMA Partnership et al. 2015; Isella et al. 2016; Fedele et al. 2017), cavities (van der Marel et al. 2016a; Dong et al. 2017), and spirals (Christiaens et al. 2014; Pérez et al. 2016; Tang et al. 2017), in the dust and gas distribution.

A large variety of mechanisms has also been invoked to produce disc substructures without assuming the presence of protoplanets. Gaps/Rings might be zonal-flows (Johansen et al. 2009), self-induced dust pile-ups due to radial migration (Gonzalez et al. 2015), pebble growth around condensation front (Zhang et al. 2015), or gradient in the viscosity at the outer edge of a dead-zone (Regály et al. 2012; Ruge et al. 2016). Local dust concentrations might be vortices induced by a large range of instabilities such the baroclinic instability (Lesur \& Papaloizou 2010) or the vertical shear instability (Nelson et al. 2013), which do not require a planet. Importantly, in the case of cavity + horseshoe, the horseshoe might not be a vortex but might be induced naturally if the disc surrounds a binary system (Ragusa et al. 2017). Finally, spirals might also be produced by the development of gravitational instabilities (Kratter \& Lodato 2016), by an external high-mass perturber undergoing a flyby (Quillen et al. 2005) or induced dynamically as a consequence of a different irradiation from the star due to a warp in the inner disc (Montesinos et al. 2016).

Due to the large range of scenarios able to produce substructures, the need of observations at high angular resolution, using different tracers, is primordial. In this work, we show ALMA observations of the protoplanetary disk around MWC 758, that we also compare with previous images obtained in near-IR scattered light by Benisty et al. (2015). The central star of the system MWC 758 is an Herbig Ae star located at a distance of $151 \pm 9 \mathrm{pc}$, based on the stellar parallax measured by the Gaia space telescope (Gaia Collaboration et al. 2016a, 2016b). This is less than the previous distance of $279_{-58}^{+94} \mathrm{pc}$ estimated from HIPPARCOS (van Leeuwen 2007). The star is surrounded by an accretion disk characterized by a complex morphology. Despite showing strong infrared excess and an accretion rate of $10^{-8} M_{\odot} \mathrm{yr}^{-1}$, the disk has a large millimeter cavity of a few tens of au in radius (Isella et al. 2010; Marino et al. 2015b). This suggests 
that the cavity might have been generated by tidal interactions with companions and not photoevaporation by the central star (Andrews et al. 2011; Owen et al. 2011; Reggiani et al. 2017). Furthermore, previous observations at radio-wavelengths have revealed the presence of asymmetries in the dust continuum emission possibly related to the accumulation of submillimeter grains in gas pressure maxima (Marino et al. 2015b). The disk also presents two spirals arms detected in near-IR scattered light (Grady et al. 2013; Benisty et al. 2015), which might be interpreted as spiral density waves launched by planet(s) of a few $M_{\text {Jup }}$ (Dong et al. 2015).

Our new observations image the MWC 758 system in both the dust continuum emission, at a wavelength of $\sim 0.88$ millimeter, and in the ${ }^{13} \mathrm{CO}$ and $\mathrm{C}^{18} \mathrm{O} J=3-2$ emission lines. The observations achieve a resolution of 0 ". $1-0$ ". 2 , or 15-30 au according to the distance of the system, which corresponds to a factor 4 of improvement with respect to previous millimeter-wave observations, and reveal unprecedented details of the disk morphology. In Section 2, we describe the acquisition of the data and the related reduction procedure, while in Section 3 we present a morphological analysis of the maps of both the continuum and the molecular emission lines. In Section 4, we constrain the physical properties of the circumstellar dust and gas by comparing the observations with radiative transfer models for the disk emission. The discussion of the results and the conclusion follow in Sections 5 and 6, respectively.

\section{Observations and Data Reduction}

We observed MWC 758 using ALMA in Cycle 3 at Band 7 as part of the project 2012.1.00725.S. Observations were performed on 2015 September 1 and 24, when 35 and 34 antennas were available, respectively. Baseline lengths ranged between $15.1 \mathrm{~m}$ and $1.6 \mathrm{~km}$ on September 1, and between $43.3 \mathrm{~m}$ and $2.3 \mathrm{~km}$ on September 24. The total on-source time spent on MWC 758 was about 58 minutes.

The ALMA correlator was configured to record dual polarization with four separate spectral windows centered at 329.34, 330.60, 341.01, and 343.01 GHz. The two first spectral windows were chosen to cover the molecular emission of $\mathrm{C}^{18} \mathrm{O}$ $J=3-2$ and ${ }^{13} \mathrm{CO} J=3-2$, with a bandwidth for each spectral windows of about $234.4 \mathrm{MHz}$. The channel width in these two spectral windows is $61.0 \mathrm{kHz}\left(0.055 \mathrm{~km} \mathrm{~s}^{-1}\right)$. The spectral resolution is twice the channel spacing since the data are Hanning smoothed. The third and fourth spectral window cover the continuum emission centered a $342 \mathrm{GHz}(\lambda \sim 0.88 \mathrm{~mm})$ with a total bandwidth of about $3.6 \mathrm{GHz}$.

The data were calibrated by the National Radio Astronomy Observatory (NRAO) staff using the CASA software package version 4.3.1 (McMullin et al. 2007). Simultaneous observations of the $183 \mathrm{GHz}$ water line with the water vapour radiometers were used to reduce atmospheric phase noise before using $\mathrm{J} 0510+180$ and $\mathrm{J} 0521+2112$ for standard complex calibration. $\mathrm{J} 0510+180$ and $\mathrm{J} 0423-0120$ were used to calibrate the frequency-dependent bandpass. The flux scale was determined with observations of $\mathrm{J} 0510+180$, for which a flux of $\approx 2.3 \mathrm{Jy}$ was adopted. We self-calibrated the dust emission and then applied the results to the line emission. We used a time interval of $30 \mathrm{~s}$ for self-calibrating the phase and in a second step the scan length $(\sim 5 \mathrm{mn})$ for the amplitude calibration.
For the final imaging step, we used different weighting parameters on the visibilities to obtain the best rendering in term of sensitivity and angular resolution for each observations. We imaged the gas emission with natural weighting. This gives to each baseline a weight inversely proportional to its associated noise, privileging the short baselines. This yields a FWHM beam size of 0 ". $23 \times 0$ ". 18 , corresponding to a spatial resolution of $34 \times 28 \mathrm{au}$, and an $\mathrm{rms}$ noise of $8 \mathrm{mJy}$ beam $^{-1}$ per channel in ${ }^{13} \mathrm{CO}$ and $11.0 \mathrm{mJy}$ beam $^{-1}$ per channel in $\mathrm{C}^{18} \mathrm{O}$. Due to its higher signal-to-noise, the dust emission is imaged with modes giving more weight to longer baselines. First, we used a Briggs parameter equal to 0 , an intermediate value, yielding a FWHM beam size of 0 " $16 \times 0$ ". 14 and an rms noise of $83 \mu \mathrm{Jy}_{\mathrm{beam}}{ }^{-1}$. We used then the super-uniform mode, with a weight proportional to the baselines length, to better detect substructures. This gives a FWHM beam size of 0 !" $12 \times 0$ !" 11 , corresponding to a spatial resolution of $18 \times 16 \mathrm{au}$, and an rms noise of $115 \mu \mathrm{Jy}$ beam $^{-1}$.

\section{Results \\ 3.1. Dust Continuum Emission}

The image of the dust continuum emission is shown in Figure 1, and was obtained adopting a Briggs weight parameter equal to 0 . The FWHM beam size is 0 ". $16 \times 0$ ". 14 (P.A. $\left.=31^{\circ} .1\right)$. The rms noise level is $83 \mu \mathrm{Jy}_{\text {beam }}{ }^{-1}$. The white cross pinpoints the center of rotation as measured from the ${ }^{13} \mathrm{CO}$ emission (see Section 3.2) and is located at R.A. = $05^{\mathrm{h}} 30^{\mathrm{m}} 27^{\mathrm{s}} .536$ and decl. $=+25^{\circ} 19^{\prime} 56^{\prime \prime} .70$, cospatial with the Herbig star position. In the same figure, the top right panel displays the deprojected intensity as a function of the distance from the disk center and the position angle (P.A.), defined as the angle east of north (i.e., toward the left). The orbital radius has been calculated by assuming an inclination of $21^{\circ}$ and a P.A. of $62^{\circ}$ for the disk. The black dashed line indicates the crest of the dust emission, which is defined as the line that connects the radial maximum of the emission for each position angle.

The dust emission is characterized by a ring structure extending between roughly 40 and 100 au and two prominent azimuthal clumps. The brightest clump has a peak intensity of $17.2 \mathrm{mJy}$ beam $^{-1}$, and is located in the northwest of the disk, at $R=81$ au and P.A. $=335^{\circ}$. The second clump has a peak intensity of $13.7 \mathrm{mJy}$ beam $^{-1}$, and is located on the southwest, at a radius of $46 \mathrm{au}$ and P.A. $=209^{\circ}$. As shown by the deprojected flux map, the dust clumps correspond to the innermost and outermost radial positions of the dust crest, whose average radial distance from the disk center is $60 \mathrm{au}$. The brightness temperature of the north and south clumps, obtained by using the inverse of the Planck function, are $14.7 \mathrm{~K}$ and $12.9 \mathrm{~K}$, respectively. These temperatures are lower by a factor $\sim 4$ than the predicted physical temperatures of the dust at the location of the clumps (see Section 4.1), therefore suggesting that the dust emission is optically thin.

The bottom panel of Figure 1 indicates the intensity along the crest of the dust emission as well as the azimuthal profile of the dust emission at the radii of the two clumps. The contrast of the north and south dust clumps relative to the average emission at the same radial position is about 4 and 2.5, respectively. 

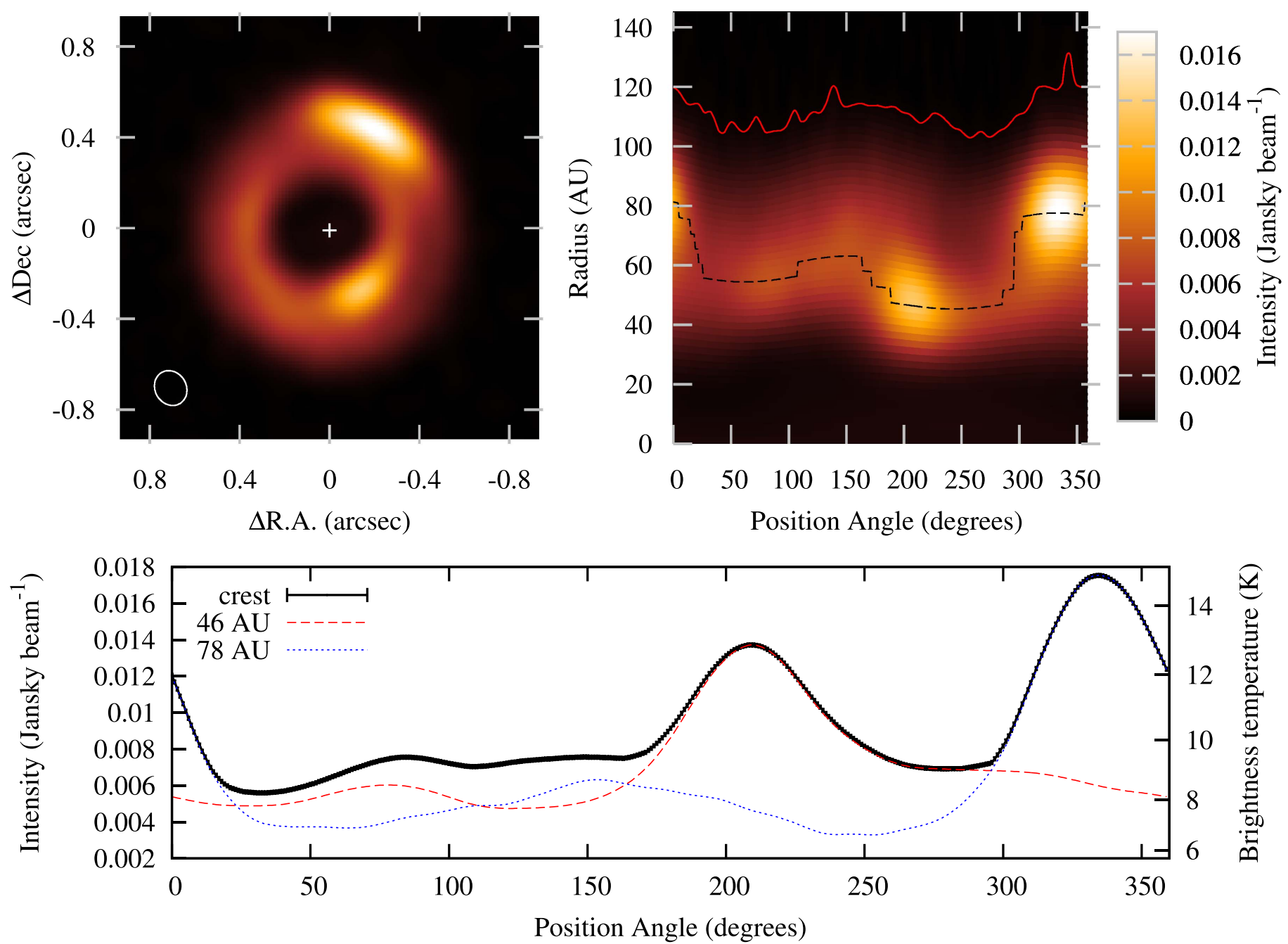

Figure 1. Top left: map of the dust continuum emission recorded at a frequency of $342 \mathrm{GHz}$. The FWHM of the synthesized beam is 0 ". $16 \times 0$ ". 14 (P.A. $=31.1$ degrees) and is indicated by the white ellipse. Top right: dust emission as a function of the orbital radius and of the position angle (P.A.) measured from the north to the east. The red solid line is the $5 \sigma$ contour, corresponding to $0.4 \mathrm{mJy}_{\text {beam }}{ }^{-1}$, and the black dashed line indicates the crest of the emission, defined as the line that connects the peaks of the emission measured at each P.A. Bottom: dust emission as a function of the P.A. The black solid line is the emission along the crest. The dashed red and blue lines are at the radii of the south and north dust clumps, respectively.

Figure 2 shows the continuum map obtained with superuniform weighting, which delivers a smaller beam size

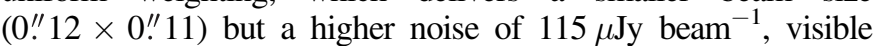
by dot-like features in the image. This new image better reveals the azimuthal morphology of the dust clumps. The peak emission is 12.3 and $10.2 \mathrm{mJy}^{\text {beam }^{-1}}$ for the north and south clump, respectively. Converted in brightness temperature, the values are, 17.2 and $15.3 \mathrm{~K}$, slightly larger than with the previous weighting parameter Briggs $=0$, indicating a better resolution, mainly radially, of the dust clumps structure. Also, the dust clumps appear to be linked to a double-ring structure, located at the same radii. Finally, the continuum emission presents also substructures that may trace faint spirals in the dust. A detailed discussion of these features and a comparison with the ${ }^{13} \mathrm{CO}$ peak emission line and previous IR observations are done in Section 4.2.

On the right panel, an inset toward the disk center shows a non-resolved source of continuum emission detected inside the cavity. The peak emission of the signal is $0.83 \mathrm{mJy}_{\text {beam }}{ }^{-1}$, about 7.2 times the noise level in the outer regions of the map. Due to the close proximity with the center of rotation of the disk, this emission probably comes from an inner disk in rotation around the Herbig Ae star. This is consistent with the spectral energy distribution of the disk that present near-IR emission, and traces warm dust close to the star (Eisner et al. 2004; Isella et al. 2008). As the signal is not resolved, this implies an outer radius $\leqslant 8$ au for the inner disk.

\section{2. ${ }^{13} \mathrm{CO}$ and $\mathrm{C}^{18} \mathrm{O} \mathrm{J}=3-2$ Emission}

The ${ }^{13} \mathrm{CO}$ and $\mathrm{C}^{18} \mathrm{O}$ emission lines were imaged using a Briggs factor of 2, to optimize the signal-to-noise ratio. The top and bottom left panels in Figure 3 show the moment 0 maps after continuum subtraction. The white cross represents the center of rotation of the disk, as derived from the ${ }^{13} \mathrm{CO}$ velocity map, shown in Figure 5. The ${ }^{13} \mathrm{CO}$ emission is more radially extended than the dust continuum, with signal detected up to about 140 au from the star. On the contrary, the radial extent of the $\mathrm{C}^{18} \mathrm{O}$ emission is about $100 \mathrm{au}$, similarly to the dust. This difference is likely due to the lower optical depth of $\mathrm{C}^{18} \mathrm{O}$ compared with ${ }^{13} \mathrm{CO}$.

The right panels in Figure 3 present the emission, the crest, and the $3 \sigma$ contours as a function of the position angle. The radius of the crest varies between 40 and 50 au for ${ }^{13} \mathrm{CO}$ and from 50 to $60 \mathrm{au}$ for $\mathrm{C}^{18} \mathrm{O}$. This suggests that the gas surface 

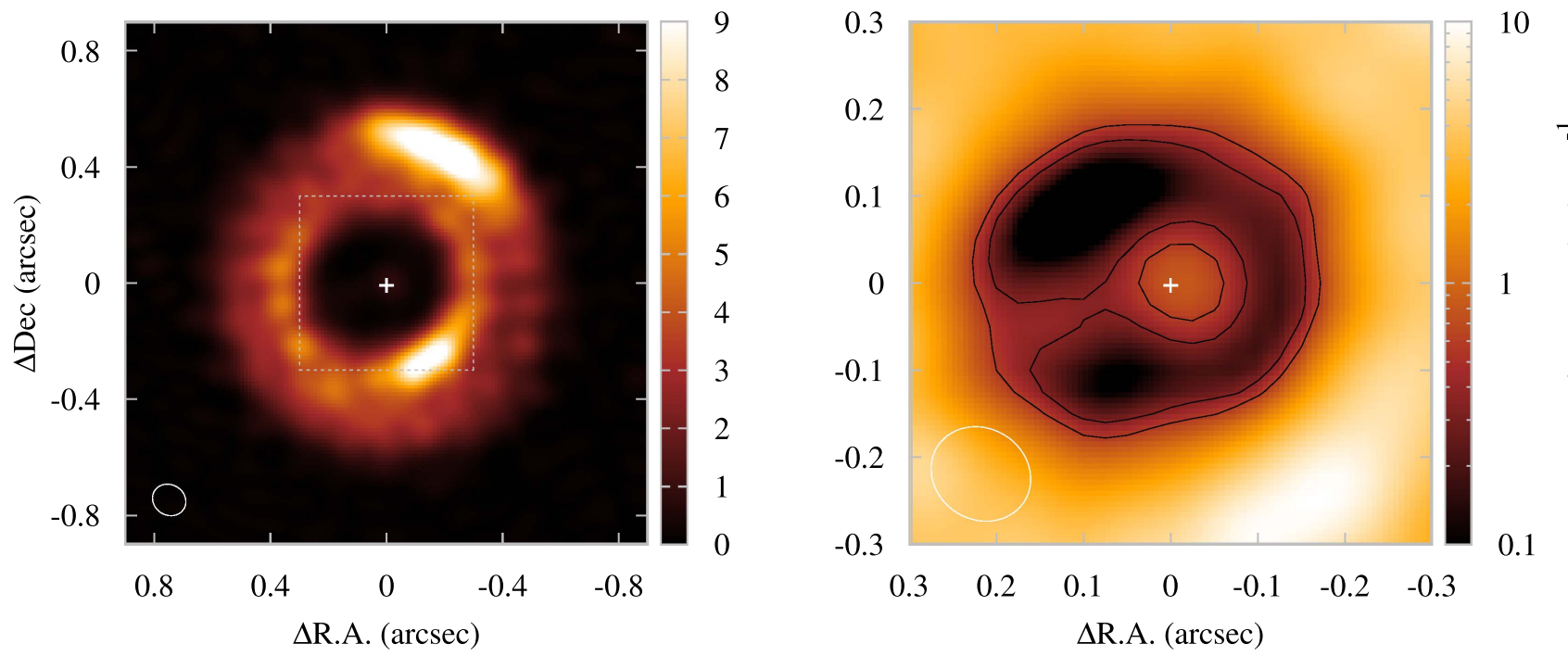

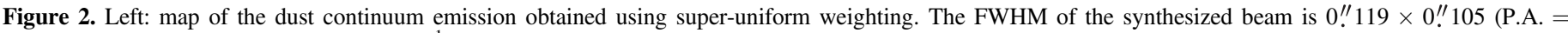

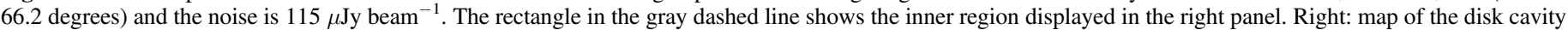

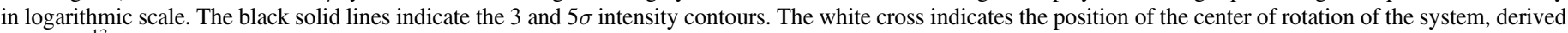
from the ${ }^{13} \mathrm{CO} J=3-2$ emission line.

density increases from the disk center to about 50-60 au. The crest radius for the two molecules is almost constant as a function of the azimuth, with variations no larger than $10 \mathrm{au}$. In particular, we do not observe any increase in the $\mathrm{CO}$ emission at the position of the two dust clumps.

The velocity map of ${ }^{13} \mathrm{CO}$ is shown in Figure 5, in Section 3.3, and indicates a position angle of $\sim 62 \pm 2^{\circ}$. Along the major axis, the northeast part (on the left) is blueshifted while the southwest part is redshifted. If the IR features observed in Grady et al. (2013) and Benisty et al. (2015) are trailing spirals, the disk is rotating clockwise and the northwest part of the disk is the closest to us. This seems confirmed by the position angle at which the emission of the two molecules reaches a maximum, between roughly $90^{\circ}$ and $180^{\circ}$, with a value of $223 \mathrm{mJy}_{\text {beam }}{ }^{-1} \mathrm{~km} \mathrm{~s}^{-1}$ at $\mathrm{PA}=125^{\circ}$ for ${ }^{13} \mathrm{CO}$, and $132 \mathrm{mJy}^{\circ}$ beam ${ }^{-1} \mathrm{~km} \mathrm{~s}^{-1}$ at $\mathrm{PA}=160^{\circ}$ for $\mathrm{C}^{18} \mathrm{O}$. If the southeast side is indeed the further half, the inner wall of the disk in this direction is directly exposed to the observer. The azimuthal minimum in gas is at a P.A. of about $240^{\circ}$, with a value 1.4 times lower than the maximum in ${ }^{13} \mathrm{CO}$ and 1.9 times the maximum in $\mathrm{C}^{18} \mathrm{O}$.

In Figure 4, we show the peak intensity maps of the ${ }^{13} \mathrm{CO}$ and $\mathrm{C}^{18} \mathrm{O}$ emission lines. The ${ }^{13} \mathrm{CO}$ peak emission is our most optically thick tracer and reveals a large spiral arm, as indicated by the increasing radius of the crest of the emission as a function of the position angle, between P.A. $=50^{\circ}$ and $200^{\circ}$. A secondary spiral arm may also be visible at P.A. $=220^{\circ}-$ $360^{\circ}$ but with less contrast with the surrounding disk. Along the crest, the brightness temperature of the emission varies between 40 and $60 \mathrm{~K}$ but shows little dependence on the orbital radius. In Section 4.1, we will show that these values of the brightness temperature are consistent with optically thick emission, with $\tau \sim 3.7$, and probably indicate variations in the temperature.

In $\mathrm{C}^{18} \mathrm{O}$, the observations present similar but less pronounced spirals. The crest of the emission, indicated by the black dashed line, is more centered at the position of $60 \mathrm{au}$, as for the integrated emission. This is probably due to the lower optical depth of this tracer, with a maximum optical depth of $\sim 0.6$ according to our model in Section 4.1. It is then less sensitive to variation in the temperature than ${ }^{13} \mathrm{CO}$ and better traces the gas surface density. This is similar to the integrated emission maps which are more optically thin than the peak emission maps, and do not present spirals.

As a note, the peak emission map was obtained without subtracting the continuum emission, that would have been resulted in underestimating the line peak brightness temperature (see the discussion in Boehler et al. 2017). However, the differences between the two methods are minor in MWC 758 due to the low optical depth of the dust.

\subsection{Disk Dynamic: An Inner Warped Disk?}

We present in Figure 5 the kinematic of the disk. The major axis of the disk has a position angle of $62^{\circ} \pm 2^{\circ}$ and the disk has a systemic velocity of $5.90 \pm 0.05 \mathrm{~km} \mathrm{~s}^{-1}$. The velocity map is globally consistent with a disk in Keplerian rotation around a central star of $1.4 \pm 0.3$ solar masses, considering an inclination of $21^{\circ}$ (Isella et al. 2010). Inside the dust cavity, however, the gas presents hints of departure from Keplerian velocity. The twist of the iso-velocity curves might reveal radial inflow of the gas or a warp linking the outer disk with a non-coplanar inner disk, where a non-resolved source of dust emission is also observed.

The panel in the right side of Figure 5 shows the ${ }^{13} \mathrm{CO}$ velocity dispersion. The velocity dispersion usually decreases with the radius due mainly to lower temperatures. It can also vary azimuthally, with a larger range of projected velocities probed along the minor axis. We nevertheless also observe a minimum along the west side of the major axis, with values of $0.3 \mathrm{~km} \mathrm{~s}^{-1}$ compared with $0.4 \mathrm{~km} \mathrm{~s}^{-1}$ along the east side. We previously pointed out in the integrated ${ }^{13} \mathrm{CO}$ and $\mathrm{C}^{18} \mathrm{O}$ emission maps an azimuthal minimum along this direction. Such diminution is consistent with an azimuthal dip of the temperature. However, it appears at a first glance surprising that the ${ }^{13} \mathrm{CO}$ peak emission, which is our most optically thick tracer, shows a bright emission along this azimuthal direction, at $\sim 40$ au, at the inner edge of the outer ring. This suggests that the azimuthal decrease in emission might be due to a shadow 

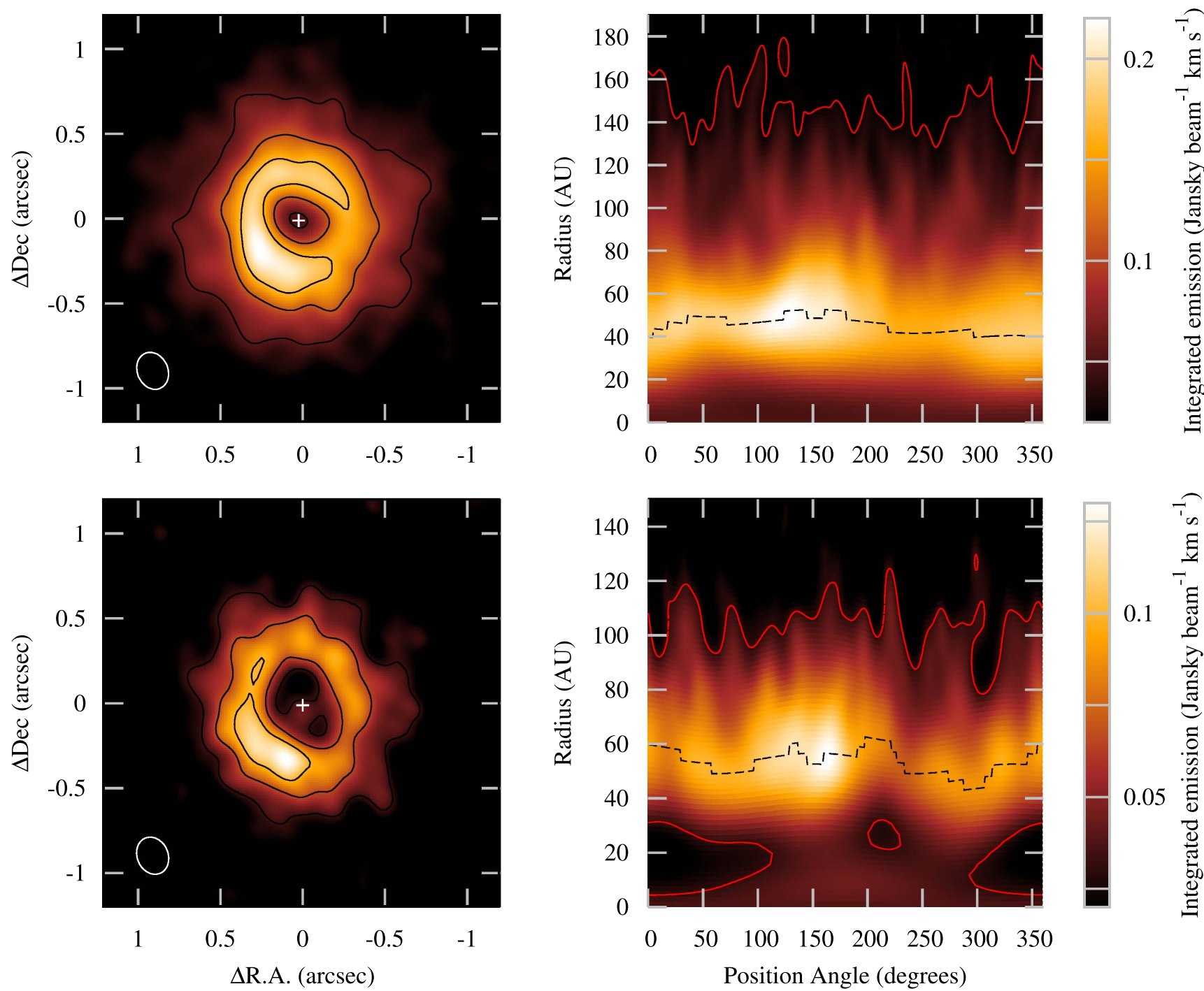

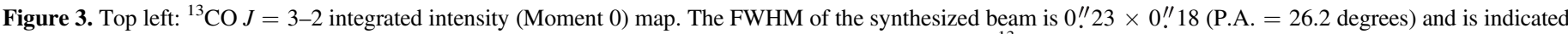

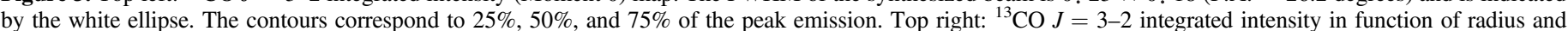

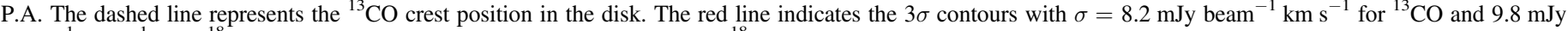
beam ${ }^{-1} \mathrm{~km} \mathrm{~s}^{-1}$ for $\mathrm{C}^{18} \mathrm{O}$. Bottom panels: same as on the top, but for the $\mathrm{C}^{18} \mathrm{O} J=3-2$ emission line.

effect from the inner edge of the outer disk, where a spiral is present and might have a higher scale height, or from an inner warped disk.

To estimate qualitatively the hypothesis of an inner warped disk, we compared our observations with the simulations of Facchini et al. (2018). They performed synthetic images of disks having a relative inclination of $30^{\circ}$ and $70^{\circ}$. The case with a moderate inclination predicts variations in the iso-velocity curves similar to what is observed in MWC 758 (i.e., their Figure 17). Also, an inner disk with a mild relative inclination will project a large shadow toward one side of the disk, but still letting an inner central region to be irradiated by the central star due to the relative geometry of the two disks (see Figures 9 and 11 in Facchini et al. 2018). Our ALMA data are then consistent with an inner mildly warped disk whose west side is the closer side. This is also supported by previous near-IR interferometric observations of Eisner et al. (2004) and Isella et al. (2008) that estimated the inclination of the inner regions to be in the range 30-40 degrees, instead of $21 \pm 2$ degrees for the outer regions (Isella et al. 2010).
Future $\mathrm{CO}$ emission line observations with a better angular resolution and sensitivity, perhaps using higher rotational lines, will help to constrain precisely the velocity structure in the cavity. This case can be compared with the disk HD 135344B which also probably has a mildly inner warped disk (Stolker et al. 2016). It is different from the binary system HD 142527, which contains a strongly warped disk, by 70 degrees (Casassus et al. 2015a), and projects two shadows in the outer regions separated azimuthally by $\sim 180$ degrees (Marino et al. 2015a; Casassus et al. 2015b; Boehler et al. 2017).

\section{Analysis}

\subsection{Modeling of the Dust and Gas Emission}

The disk around MWC 758 has a complex morphology, characterized by dust clumps and spirals surrounding a large cavity. A complete modeling of the 3D structure of the disk would be very difficult. We performed instead a study which focuses on three particular azimuthal directions, as shown in Figure 6 . The emission coming from the red area, will be used to 

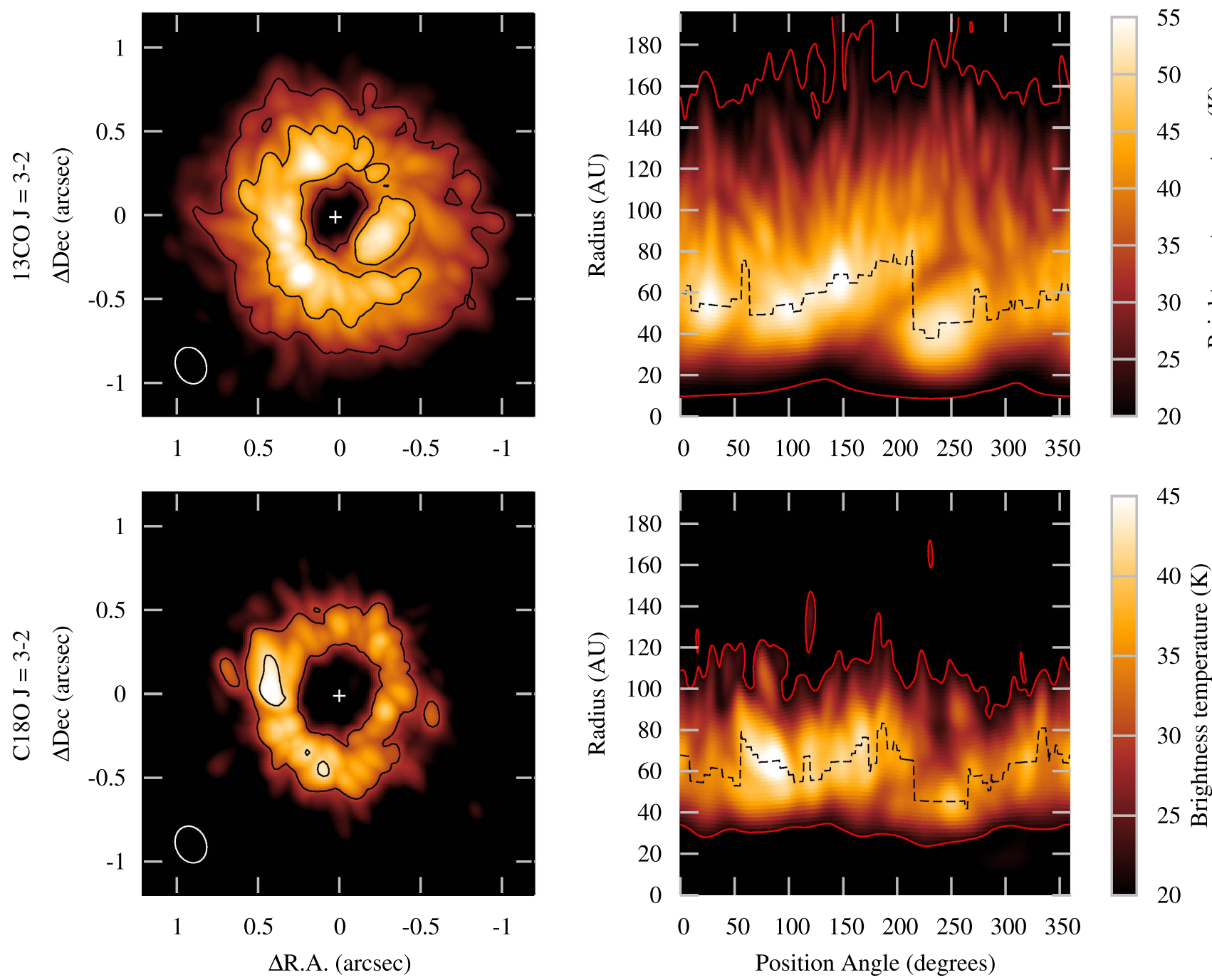

Figure 4. Left: maps of the ${ }^{13} \mathrm{CO}$ and $\mathrm{C}^{18} \mathrm{O} J=3-2$ peak emission line in units of the brightness temperature. The contours are plotted at $30 \mathrm{~K}$ and $42 \mathrm{~K}$. Right: peak emission line as a function of the azimuth and of the radial distance. The red line indicates the $3 \sigma$ contours with $\sigma=8.2 \mathrm{mJy}^{-} \mathrm{beam}^{-1}$ for ${ }^{13} \mathrm{CO}$ and $10.2 \mathrm{mJy}$ beam $^{-1}$ for $\mathrm{C}^{18} \mathrm{O}$, or $17.3 \mathrm{~K}$ and $19.0 \mathrm{~K}$, respectively, by using the inverse of the Planck function.

characterize the underlying rings, while we will study the variations in dust and gas surface densities along the north and south dust clumps, inside the blue and green areas, respectively.

The model discussed below follows the analysis presented in Boehler et al. (2017). Following the observations of the dust with super-uniform weighting, we assume a surface density structure for the dust and the gas represented by the sum of two Gaussians, using the formula

$$
\begin{aligned}
\Sigma(r)= & \Sigma_{1} \exp \left(-\left(\frac{r-R_{1}}{w_{1}}\right)^{2}\right) \\
& +\Sigma_{2} \exp \left(-\left(\frac{r-R_{2}}{w_{2}}\right)^{2}\right),
\end{aligned}
$$

with $R 1$ and $R_{2}$, the radial positions of the two Gaussians, $\Sigma_{1}$ and $\Sigma_{2}$ their surface density, and $\mathrm{w} 1$ and $w_{2}$, their half-widths. The dust surface density is then described by using 6 free parameters. For the gas, less spatially resolved, we generally only use 4 free parameters, fixing the radial location of the two Gaussians at the same position as for the dust. However, in one case, along the south clump, we had to modify the radial position of the inner Gaussian on a less eccentric position to better represent the gas emission. Vertically, the gas is assumed in hydrostatic equilibrium, at the midplane temperature, and the dust vertically mixed with the gas. We also tested two different degrees of dust settling, with the dust vertical scale height reduced by a factor of 5 and 10, but only negligible changes were observed between the models.

The temperature in the disk is determined by carrying out a two-dimensional $(r, z)$ radiative transfer using the code RADMC-3D (Dullemond et al. 2012). The central star is an Herbig A5e star, with a temperature at the surface of $8130 \mathrm{~K}$ (van den Ancker \& de Winter 1998). Taking into account the new estimated distance of $151 \mathrm{pc}$, the intrinsic luminosity is now $15.3 L_{\odot}$ with a radius for the star of $2.0 R_{\odot}$. The mass, deduced from Keplerian rotation, is $1.4 \pm 0.3 M_{\odot}$. The stellar emission is mainly absorbed in protoplanetary disks by $\mu \mathrm{m}$-size grains, which are expected to be well coupled to the gas. We then assume for the radiative transfer the standard gas-to-dust ratio of 100 , which corresponds to an opacity of about $6 \mathrm{~cm}^{2} \mathrm{~g}^{-1}$ of gas, in the frequency range $0.1-1 \mu \mathrm{m}$, at which most of the stellar energy is absorbed. The dust grains responsible of the dust 

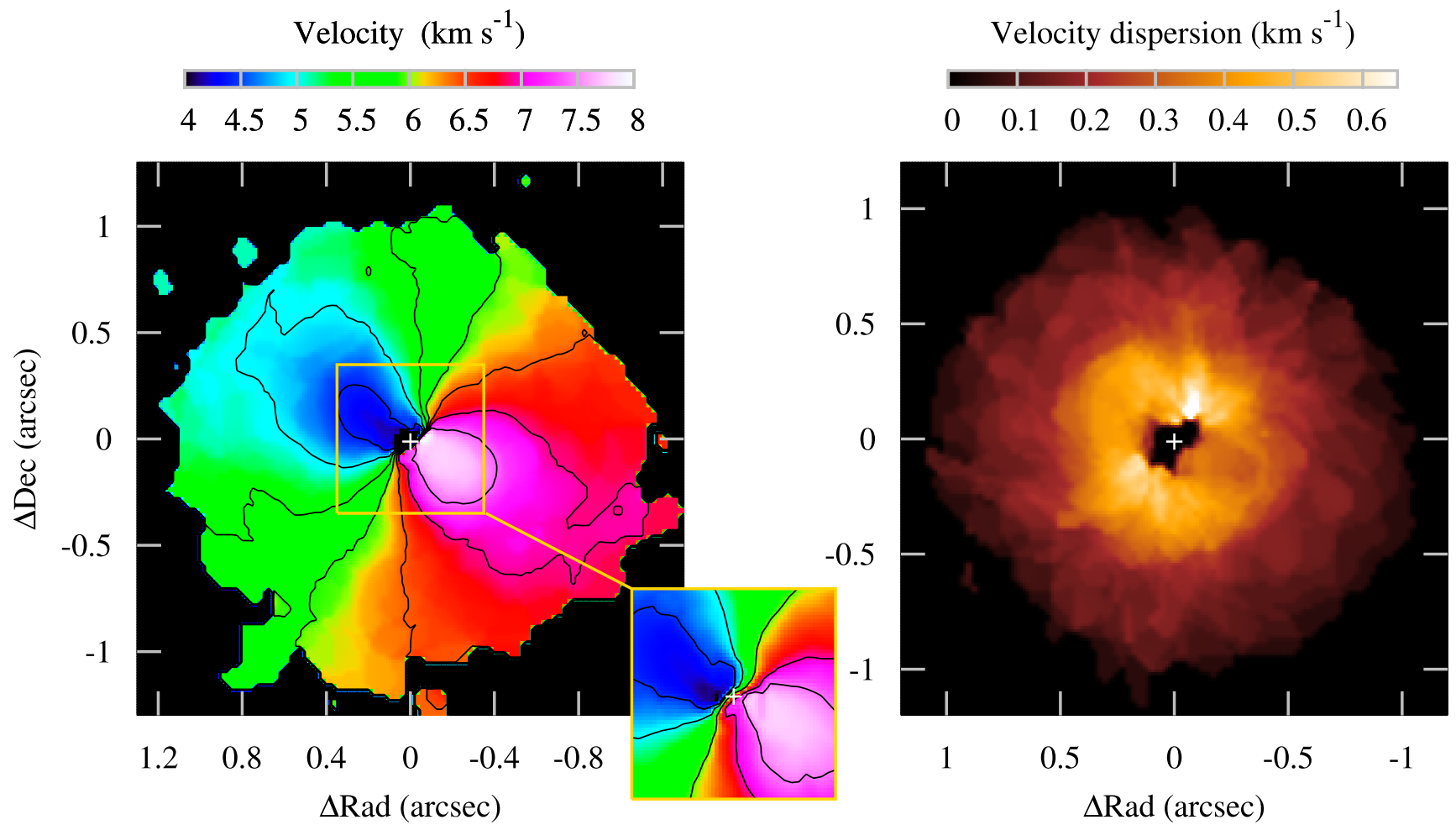

Figure 5. Left: map of the velocity centroid for the ${ }^{13} \mathrm{CO} J=3-2$ line plotted between 4 and $8 \mathrm{~km} \mathrm{~s}^{-1}$. The systemic velocity is $5.90 \mathrm{~km} \mathrm{~s}$ and the contours are displayed from 4.40 to $7.40 \mathrm{~km} \mathrm{~s}^{-1}$ by paths of $0.5 \mathrm{~km} \mathrm{~s}^{-1}$. The inset shows the inner regions of the velocity map, taking into account all the channels above $3 \sigma$ (with $\sigma=8 \mathrm{mJy}^{\text {beam }}{ }^{-1}$ ) instead of $5 \sigma$ for the larger map. Right: map of the velocity dispersion in ${ }^{13} \mathrm{CO}$ plotted between 0 and $0.65 \mathrm{~km} \mathrm{~s}^{-1}$.

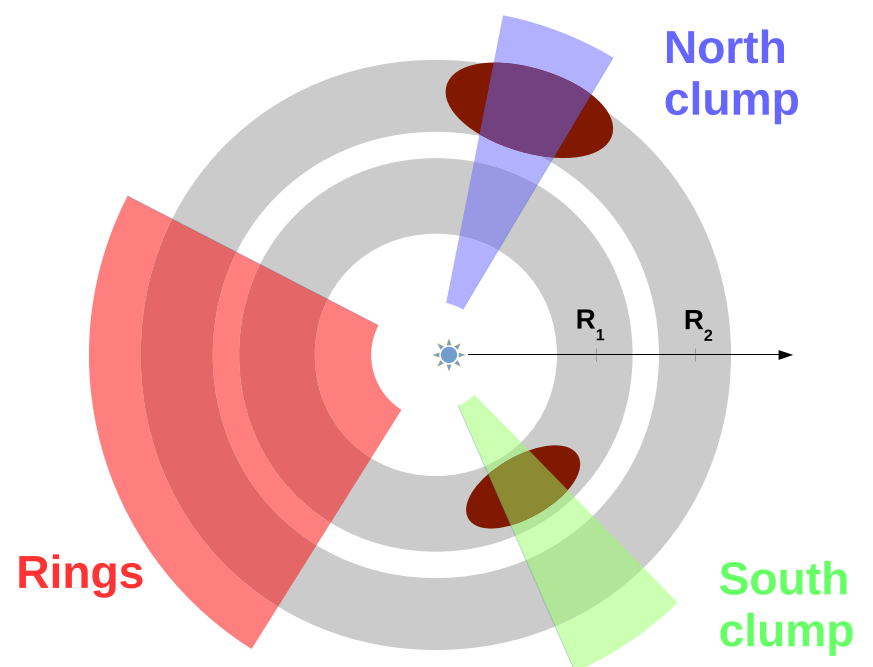

Figure 6. Sketch of the MWC 758 system with the three azimuthal directions used for the disk modeling. The underlying rings are modeled using the emission in the angular range $75^{\circ}-150^{\circ}$ represented by the red area. In green, the model across the south dust clump in the angular range $200^{\circ}$ and $220^{\circ}$, and in blue the model across the north dust clump in the angular range $325^{\circ}-345^{\circ}$.

emission at millimeter wavelength are represented by a grain size distribution following a power law of exponent -3.5 between $0.05 \mu \mathrm{m}$ and $1 \mathrm{~mm}$, and a chemical composition corresponding to the solar elemental abundance (Anders \& Grevesse 1989). These dust properties were previously used in Boehler et al. (2017) and yields an opacity at 0.88 millimeter of $2.9 \mathrm{~cm}^{2} \mathrm{~g}^{-1}$. The fractional abundances of ${ }^{13} \mathrm{CO}$ and $\mathrm{C}^{18} \mathrm{O}$, with respect to molecular hydrogen, are assumed to be $9 \times 10^{-7}$, and $1.35 \times 10^{-7}$, respectively (Qi et al. 2011).
Table 1

Density Prescription for the Gaussians Describing the Dust and Gas Surface Density

\begin{tabular}{lcccccc}
\hline \hline & $\begin{array}{c}\Sigma_{1} \\
\left(\mathrm{~cm}^{2} \mathrm{~g}^{-1}\right)\end{array}$ & $\begin{array}{c}R_{1} \\
(\mathrm{au})\end{array}$ & $\begin{array}{c}w_{1} \\
(\mathrm{au})\end{array}$ & $\begin{array}{c}\Sigma_{2} \\
\left(\mathrm{~cm}^{2} \mathrm{~g}^{-1}\right)\end{array}$ & $\begin{array}{c}R_{2} \\
(\mathrm{au})\end{array}$ & $\begin{array}{c}w_{2} \\
(\mathrm{au})\end{array}$ \\
\hline Ring (P.A. $\left.=75^{\circ}-150^{\circ}\right)$ & & & & & \\
\hline Dust & 0.040 & 57 & 8 & 0.036 & 81 & 12 \\
Gas & 1.3 & 57 & 17 & 0.9 & 81 & 30 \\
\hline South Clump (P.A. $\left.=200^{\circ}-220^{\circ}\right)$ & & & & \\
\hline Dust & 0.102 & 47 & 5 & 0.030 & 78 & 18 \\
Gas & 0.9 & 53 & 17 & 0.9 & 78 & 24 \\
\hline North Clump (P.A. $\left.=325^{\circ}-345^{\circ}\right)$ & & & & \\
\hline Dust & 0.027 & 56 & 12 & 0.24 & 82 & 9 \\
Gas & 1.3 & 56 & 18 & 1.0 & 82 & 28 \\
\hline
\end{tabular}

The radial profiles of the dust continuum emission are displayed in the left panels of Figure 7 and the integrated emission of the ${ }^{13} \mathrm{CO}$ and $\mathrm{C}^{18} \mathrm{O} J=3-2$ in the right panels, from top to bottom for each of the three sectors presented in Figure 6. We use the integrated emission to fit the gas surface density distribution, as its optical depth is lower than for the peak emission, and less sensitive to variations at the disk surface, such than spirals, that are not taken into account in our modeling. At a first glance, we observe large azimuthal variations in the dust emission while the ${ }^{13} \mathrm{CO}$ and $\mathrm{C}^{18} \mathrm{O}$ integrated emissions are more constant with the position angle.

The models that best fit the data are represented by dotted lines and were found manually. They generally well recover the disk emission. A summary of the model parameters that give 

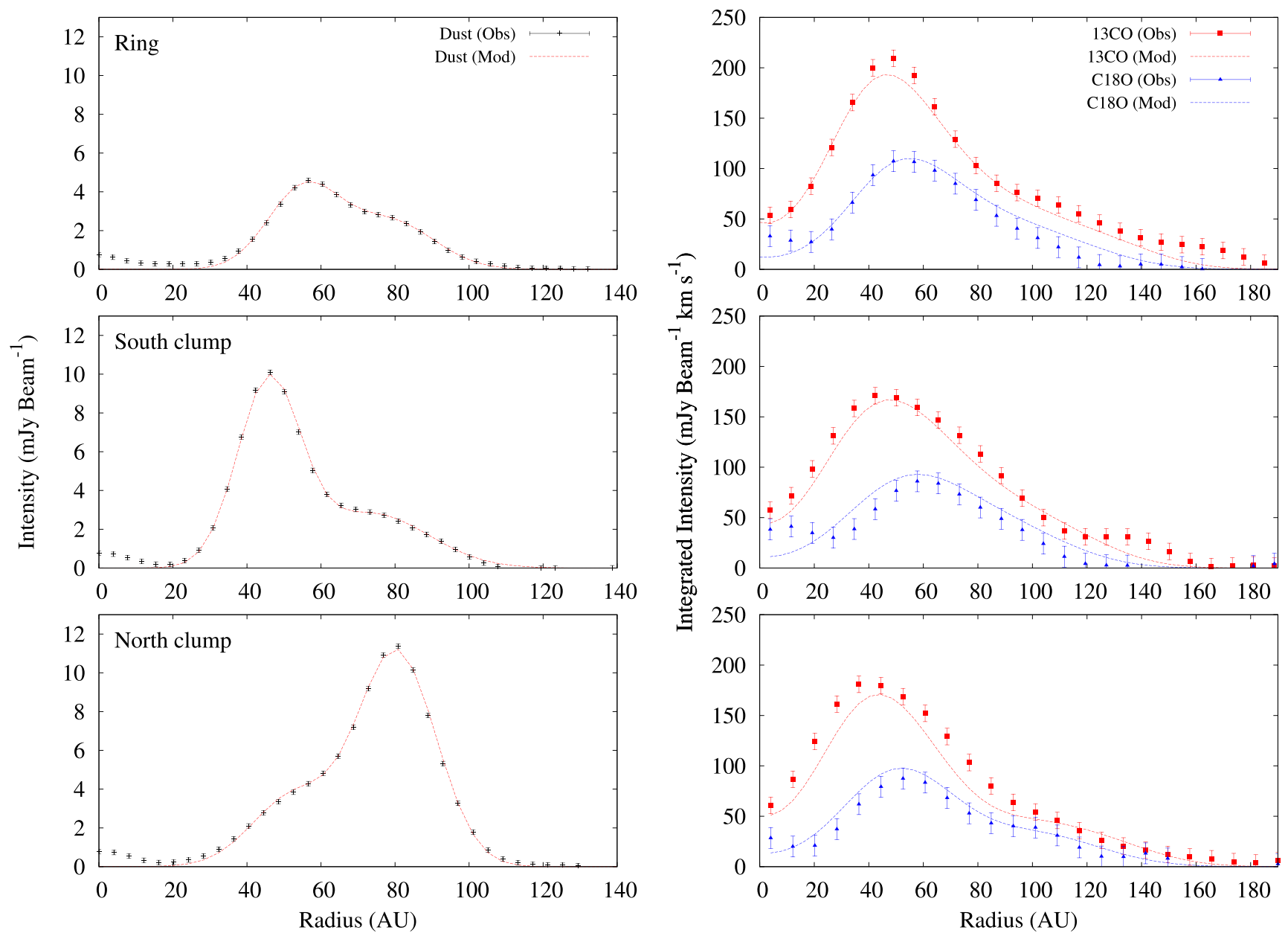

Figure 7. Left: dust continuum emission obtained with super-uniform weighting in function of the radial distance (top: P.A. $=75^{\circ}-150^{\circ}$, middle: P.A. $=200^{\circ}-220^{\circ}$, and bottom: P.A. $=325^{\circ}-345^{\circ}$ ). The observations and their associated error bars are in black, while the models are represented by red dashed lines. Right: ${ }^{13} \mathrm{CO}$ and $\mathrm{C}^{18} \mathrm{O} J=3-2$ integrated emission lines (Mom 0 ). The observations are represented by a red square for ${ }^{13} \mathrm{CO}$ and a blue triangle for $\mathrm{C}^{18} \mathrm{O}$, with their error bars, and the models are indicated by dashed lines.

the dust and gas surface densities are provided in Table 1 and in Figure 8. Figure 8 also shows the radial evolution of the dustto-gas ratio. On the left panels of Figure 7, the dust emission, optically thin, presents a double-ring structure. The two rings are located at 57 and 81 au and have a comparable surface density of $\sim 0.4 \mathrm{~g} \mathrm{~cm}^{-2}$. In comparison, the two dust clumps are on more eccentric positions, with the south clump at 47 au and the north clump at $82 \mathrm{au}$, and have surface densities of about 2.5 and 6.5 times, respectively, the values encountered in the underlying rings.

The gas has small azimuthal variations in the surface density structure. As we consider that the small grains follow the gas, the temperature of the disk is almost invariant azimuthally. The temperature along the double-ring structure is given in Figure 9. Only the gas surface density along the south dust clump appears slightly smaller. This area is located at the azimuthal minimum in the ${ }^{13} \mathrm{CO}$ and $\mathrm{C}^{18} \mathrm{O}$ integrated emission. As mentioned in Section 3.3, this region might in fact be in the shadow projected by the inner regions of the disk. In this case, the temperature would be locally lower and inversely, the gas surface density higher, more similar to the value encountered at other polar angles.

The gas distribution is also more extended radially than the large dust grains distribution, both in the cavity and in the outward direction. The lower resolution obtained for the gas of $\sim 0$ !' 2 about twice larger than in the dust, and its lower signal-to-noise, does not allow to probe as efficiently gas substructures. Therefore, we are not able to observe if the double-ring structure also exists in the gas distribution. We also do not find evidence of gas concentration cospatial with the dust clumps. This leads to an increase of the dust-to-gas ratio in the dust clumps to a value of about 0.1, shown in the bottom panel of Figure 8, 10 times the usual standard value. As a simple estimation of our precision, $\mathrm{C}^{18} \mathrm{O}$, our best tracer of the gas density, has maximum values of about $100 \mathrm{mJy}_{\text {beam }}^{-1} \mathrm{~km} \mathrm{~s}^{-1}$, about 10 times larger than the rms noise. The precision for the gas density at $3 \sigma$ is then known at best at $\pm 30 \%$.

Considering an average temperature of $60 \mathrm{~K}$ at a distance of $60 \mathrm{au}$, where the gas density reaches its maximum, we find optical depths of about $\sim 3.7$ and 0.6 at the center of the ${ }^{13} \mathrm{CO}$ and $\mathrm{C}^{18} \mathrm{O} J=3-2$ emission lines, respectively. By comparison, the dust has a maximum optical depth of about 0.12 in the underlying double disk structure, but which raises up to 0.7 in the north dust clump. Finally, if we consider that the gas and the dust distribution in the disk are represented azimuthally at $70 \%$ by the red sector, and at $15 \%$ each by the sectors along the dust clumps, the gas mass in the disk is about $1.3 \times 10^{-3} M_{\odot}$ and the dust mass about $3.0 \times 10^{-5} M_{\odot}$. 

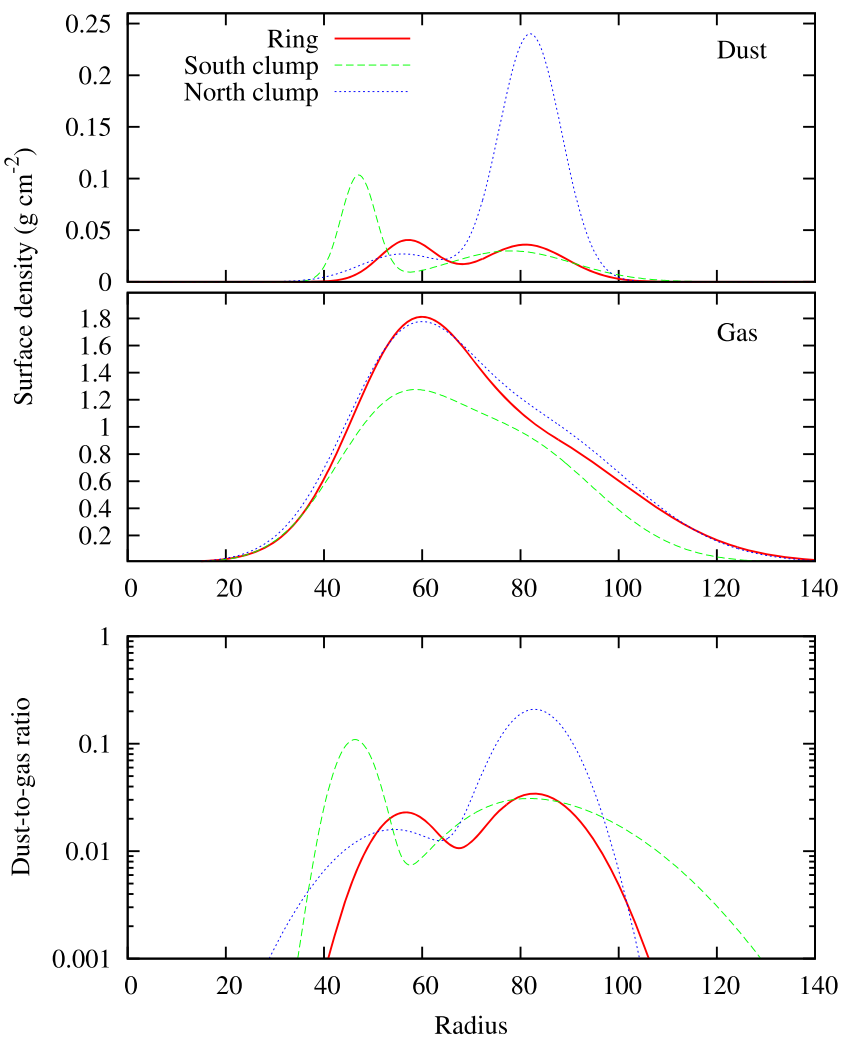

Figure 8. Top and middle panels: dust and gas surface densities across the rings $\left(\right.$ P.A. $\left.=75^{\circ}-150^{\circ}\right)$, the south dust concentration $\left(\right.$ P.A $=200^{\circ}-220^{\circ}$ ), and across the north dust concentration (P.A. $=325^{\circ}-345^{\circ}$ ). The bottom panel indicates the dust-to-gas ratio for the same three position angles.

\subsection{Comparison of the Dust and ${ }^{13}$ CO Peak Emission with the IR Scattered Light}

The near-IR emission is expected to be optically thick and to be scattered efficiently by small dust grains of a few $\mu \mathrm{m}$ in protoplanetary disks. The polarized emission therefore traces fluctuations of the disk surface and has been used in observations to detect spirals or gap structures. The observations were made by Grady et al. (2013) using the Subaru telescope in near-IR via the $H$ Band $(1.65 \mu \mathrm{m})$ in polarized emission and the $K \mathrm{~s}$ Band $(2.15 \mu \mathrm{m})$ in direct imaging. They detected 2 spirals, but with a larger contrast for the spiral starting on the east side of the star, and wrapping around the disk toward the south (SE spiral). Benisty et al. (2015) redetected the spirals with VLT/SPHERE in scattered light in $Y$ Band $(1.04 \mu \mathrm{m})$ giving further insights on the disk surface. Here we propose to compare the observations of Benisty et al. (2015) with our ALMA data to have a 3D view, of the surface and midplane, of the disk around MWC 758.

In Figure 10, we compare the near-IR observations of Benisty et al. (2015) with the ${ }^{13} \mathrm{CO}$ peak emission. The ${ }^{13} \mathrm{CO}$ peak emission is optically thick in the outer disk, with $\tau \sim 2.5$ and therefore is particularly sensitive to the disk temperature. We observe a clear correlation between the ${ }^{13} \mathrm{CO}$ peak emission and the near-IR features, especially for the southeast spiral, suggesting that the spirals observed in near-IR are coincident with a local increase in temperature. The correlation with the northwest spiral is less evident. The spiral is mainly located at a smaller radius of $40-50$ au where the ${ }^{13} \mathrm{CO}$ peak emission is more optically thin and depends also on the gas

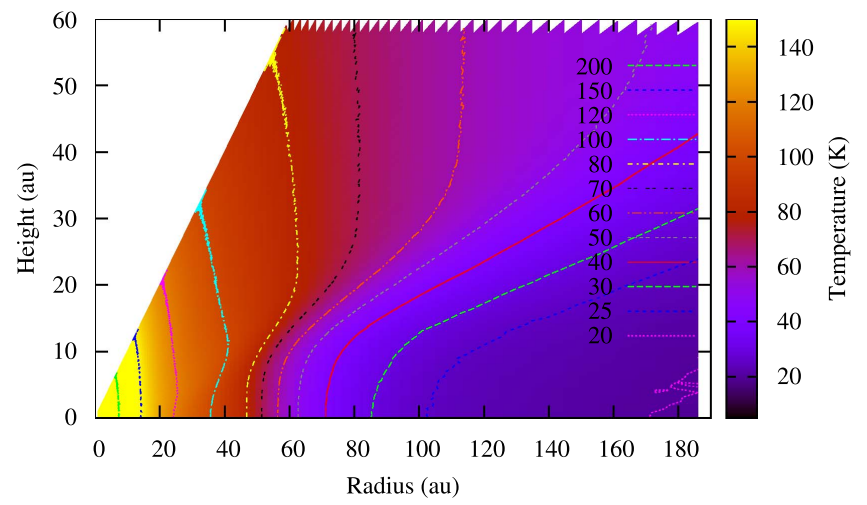

Figure 9. Temperature in the disk at P.A. between $75^{\circ}$ and $150^{\circ}$.

surface density, which increases up to $60 \mathrm{au}$. Also, in the previous IR observations of Grady et al. (2013) and Benisty et al. (2015), the northwest spiral was less pronounced or azimuthally extended, suggesting a spiral less irradiated by the central star due to shadows cast from the inner regions of the disk or a lower enhancement in density and/or vertical elevation. Using a linear regression method, we fitted the position of the IR radial maxima by spirals, with a constant pitch angle, described by the following equation:

$$
R=R_{0} \exp \left[\tan (\alpha)\left(\theta-\theta_{0}\right)\right]
$$

with $\alpha$ the pitch angle, and $R_{0}$ the radial distance of the spiral at the azimuthal angle $\theta_{0}$. These fits are represented by dashed black lines in the right panel of Figure 10. The southeast spiral, fit at P.A. between $50^{\circ}$ and $200^{\circ}$, has a pitch angle of 12.7 and is located at $R_{0}=43.0$ au at P.A. $=50^{\circ}$. The northwest spiral, fit between $230^{\circ}$ and $340^{\circ}$, features a similar pitch angle of $12^{\circ} .9$, starting at the radial distance $R_{0}=33.0 \mathrm{au}$ at P.A. $=230^{\circ}$. The angular separation between the two spirals is of about $180^{\circ}$, only varying slightly in the outer disk. There is no similar spiral-like components in the integrated emission of ${ }^{13} \mathrm{CO}$ and $\mathrm{C}^{18} \mathrm{O}$, which are more optically thin and probe essentially the gas surface density.

We also compared the position of the infrared spirals with the dust emission obtained using super-uniform weighting in Figure 11. The fit of the spirals in near-IR scattered light is represented in blue solid lines and their extrapolation toward larger radii in blue dotted lines. In addition to the double-ring structure, the dust emission reveals two faint spirals located at a slightly larger radius than the near-IR spirals (Shen et al. 2017, in preparation). Also, similarly to the near-IR observations, the pitch angle of the northwest spiral in the dust emission seems to increase at the proximity of the north dust clump.

As already noticed by Benisty et al. (2015), and more recently by Reggiani et al. (2017) using the Keck telescope, the IR scattered light presents two other features in the outer ring, designated 3 and 4 in the right panel of Figure 11. They do not trace large spirals. The feature 3 has a negative pitch angle, and both have a very limited azimuthal extent. Reggiani et al. (2017) proposed that the north IR arc was possibly tracing the spiral 1, but in the bottom side of the disk. Our new observations show however that these two features are located at, and likely trace, the inner edge of the two dust clumps. Indeed, the local enhancement in dust surface density must increase locally the absorption and scattering of the stellar 

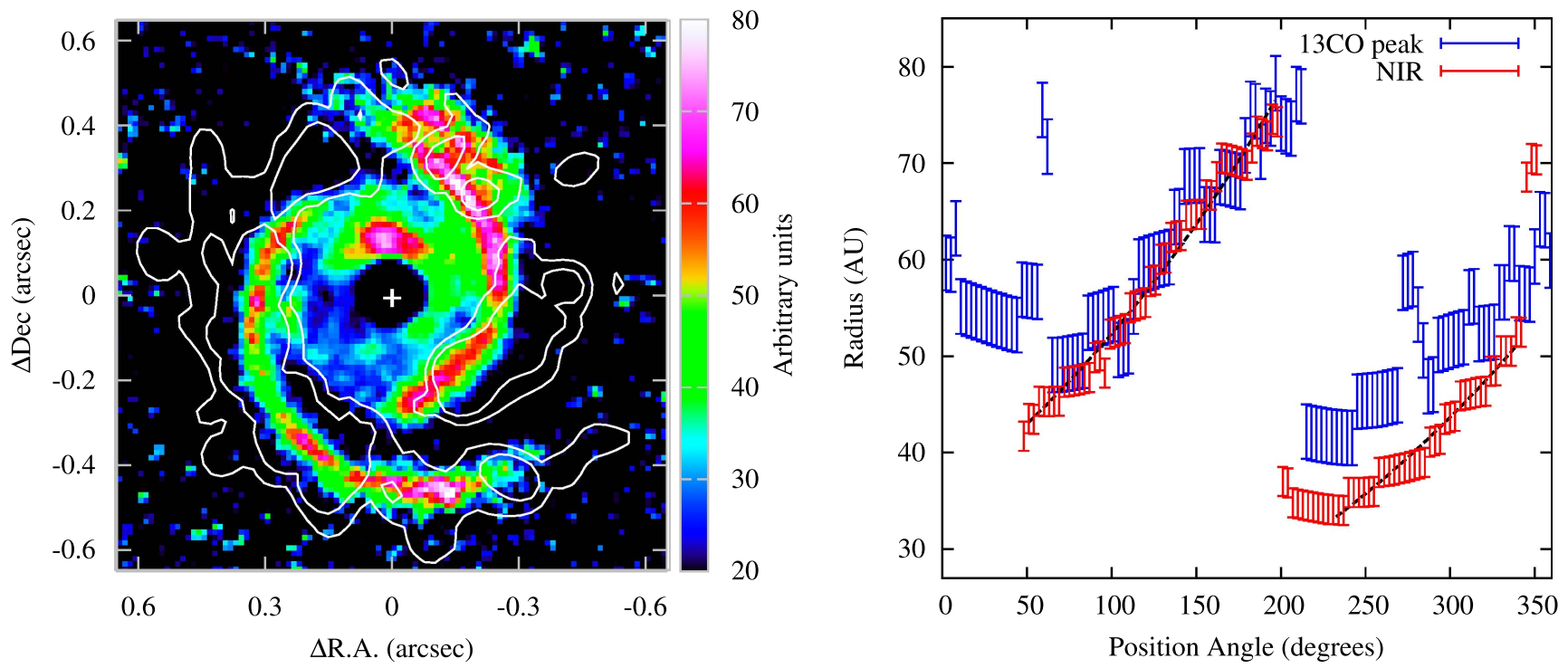

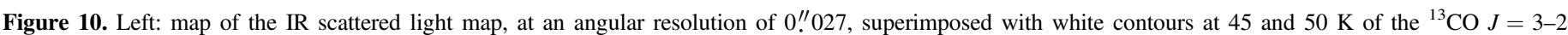

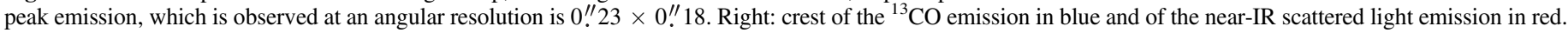
The fit of the near-IR spirals is represented by black dashed lines.

irradiation at the inner edge of the dust clumps. This may then also rise up the temperature and the disk scale height, unveiling even more the two near-IR features.

\section{Discussion}

\subsection{Structure of the Disk}

The disk around the Herbig star MWC 758 has a complex morphology which comprises a cavity, two rings, two large spirals and two dust clumps. According to our models, the disk, with a mass of about $1.3 \times 10^{-3} M_{\odot}$, is not massive enough to be gravitationally unstable (Kratter \& Lodato 2016). One explanation for such a complex structure is the presence of planet(s) which can interact with the protoplanetary disk and create features (annuli, spirals, or vortices) which can be more easily detected than the planets themselves (Kley \& Nelson 2012; Baruteau et al. 2014).

We observe in the continuum emission two large dust clumps which have an elongated structure and an azimuthal/ radial aspect ratio of approximately five at our angular resolution. To our knowledge, MWC 758 is the only disk with V1247 Orionis, observed by Kraus et al. (2017), which features two dust clumps at two different radial distances. These structures are associated with azimuthal extensions dividing the disk in two rings, located at a distance from the star of about 57 and 81 au. By modeling the dust and gas emission, we conclude that the gas distribution is more diffused than the distribution of the large dust grains. Millimeter grains are gathered in compact regions with surface densities larger by a factor of 2.5 and 6.5 in the south and north dust clumps, respectively, compared with values encountered at other azimuthal angles. We do not detect increases of the gas surface density at the position of the dust clumps, what suggests a local augmentation of the dust-to-gas ratio, up to a value of $\sim 0.1$.

The system MWC 758 is also famous for its two large and sharp spirals detected in near-IR scattered light by Grady et al. (2013), Benisty et al. (2015) and more recently by Reggiani et al. (2017). These spirals feature a large pitch angle of $\sim 13^{\circ}$ and are also observed with ALMA using the optically thick ${ }^{13} \mathrm{CO} \quad J=3-2$ peak emission and the optically thin continuum dust emission. This is the first time that spirals in protoplanetary disks are visible with these three tracers. The bright southeast spiral is observed at the same radius in IR and in ${ }^{13} \mathrm{CO}$. The IR scattered light traces the inner side of the spiral shock front at the disk surface, where the stellar emission is absorbed and locally heats the disk. In comparison, the spirals observed with the dust emission show fainter spiral structures and are located at a slightly larger radius, by a few au. The difference in the radial location might come from the width of the spirals, as we expect that the dust emission traces mainly the central position of the spirals. It can also be caused by the vertical propagation of the spirals which is expected to curl toward the central star at the disk surface instead of being perpendicular to the disk midplane (Zhu et al. 2015).

The northeast spiral is less pronounced in the ${ }^{13} \mathrm{CO}$ peak emission and (less extended azimuthally) in IR scattered light (Grady et al. 2013) than its southwest counterpart. It is possible that this spiral presents a lower scale height and/or density enhancement. We propose also that this might be due to a shadow cast by the inner regions of the disk. We detect a non-resolved source of continuum emission at the central star position, associated with a twist in the iso-velocity curves. Additionally, we detect an azimuthal minimum along the west direction in the ${ }^{13} \mathrm{CO}$ and $\mathrm{C}^{18} \mathrm{O}$ integrated emission and in the velocity dispersion, which supports the hypothesis of a mildly warped inner disk.

\subsection{Possible Scenarios}

The origin of the spirals in MWC 758 has been under debate since their discovery by Grady et al. (2013), and our ALMA observations also show the presence of two dust clumps and a dark ring, surrounding a large cavity. The south dust clump is located at the outer edge of the cavity, at $47 \mathrm{au}$, and the north dust clump, at the outer edge of the dark ring, at 82 au. These regions present large gas surface density gradients where Rossby-wave instabilities (RWIs) are thought to develop and trigger vortices. The two dust clumps are also cospatial with the 

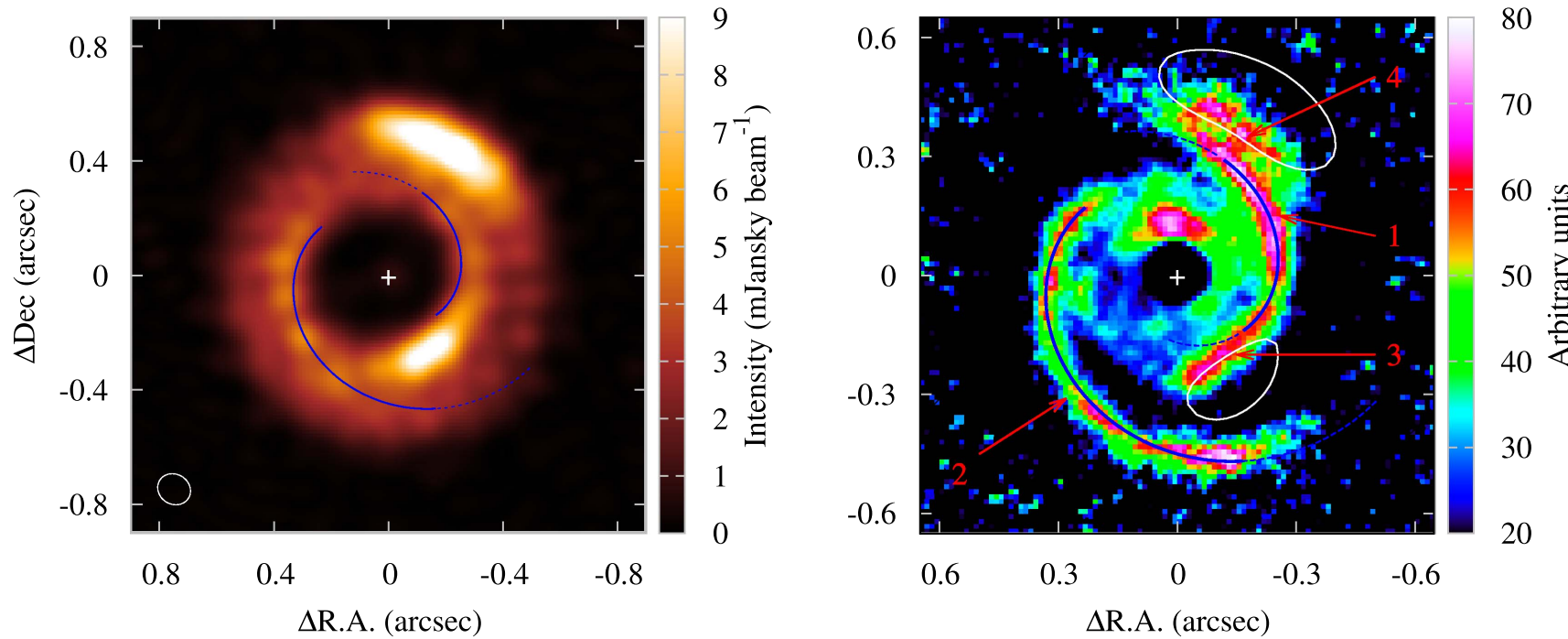

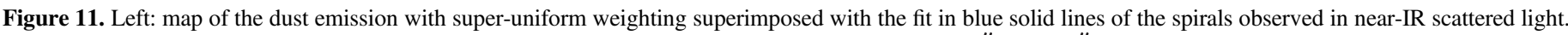

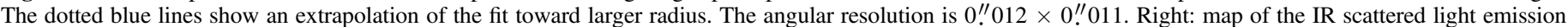

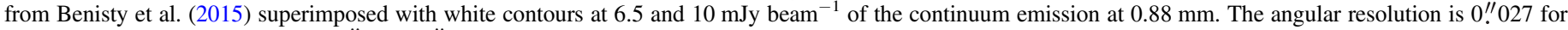

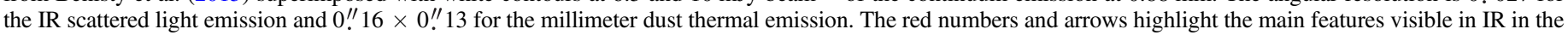
outer ring.

two azimuthal maxima detected in the continuum emission by Marino et al. (2015b) with the VLA at $33 \mathrm{GHz}$. This suggests that large dust grains are trapped into gas pressure maxima through aerodynamical drag, as already proposed by Marino et al. (2015b) for the north dust clump. Small local variations of $20 \%-30 \%$ in the gas might not be visible in our observations but still be able to accrete efficiently dust particles (Pinilla et al. 2012).

Many disks, like IRS 48 (van der Marel et al. 2013), AB Aur (Tang et al. 2017), SAO 206462 and SR 21 (Pérez et al. 2014) among others, have shown large dust asymmetries that may also suit this scenario. As for MWC 758, most of these disks comprise a large cavity, which might be open by a stellar companion or planet(s). The probable detection by Reggiani et al. (2017) of a planet of $0.5-5 M_{\text {Jup }}$ inside the cavity supports this idea. If the companion is massive enough, simulations of Ragusa et al. (2017) have shown that it can also produce a large azimuthal variation of the gas surface density. This might be the case for the binary system HD 142527, which presents a stellar companion of about $0.25 M_{\odot}$ and a large azimuthal ratio of $\sim 3.75$ in the gas surface density (Muto et al. 2015; Boehler et al. 2017). This effect is however likely negligible in MWC 758 which has a low planet-to-star mass ratio, of about 0.001 , and presents only moderate azimuthal variations of the gas surface density.

A scenario that might explain the complex structure of the outer regions of the MWC 758 system is the presence of a large planet at $R \geqslant 100$ au. 3D hydrodynamical simulations of Zhu et al. (2015) and Dong et al. (2015) have shown that a massive planet of a few Jupiter mass in the outer regions would produce a pair of inner spiral arms, whose brightness and large pitch angle are compatible with the near-IR observations. Recent simulations of Bae et al. (2017) have also shown that, for a disk with low viscosity (i.e., $\alpha \sim 10^{-4}$ ), a massive planet can also produce a secondary gap in the inner regions of the planet orbit. This gap is linked to the secondary spiral and is located at approximatively $0.5-0.6$ the orbital radius of the planet. This is consistent with the observation of the dark ring located between the two dust clumps. Such planet can also produce a tertiary spiral that might have been observed in the cavity of the disk by Reggiani et al. (2017). However, this hypothetical massive planet has not been detected yet in the IR observations of Grady et al. (2013) and Reggiani et al. (2017).

There are also processes that can explain part of the observations without the need of a planet in the outer regions (at $R \geqslant 100$ au.) A simple explanation for the dark ring is the presence of a supplementary planet of moderate mass inside the ring. It has also been proposed by Lobo Gomes et al. (2015) that a first vortex, formed at the edge of the central cavity, might accrete matter and cleans the orbit at a slightly larger radius. This process can then create a ring and be at the origin. Nevertheless, these hypotheses alone do not explain the presence of the spirals. Montesinos et al. (2016) suggested that spirals might be induced dynamically as a consequence of a different irradiation from the star due to a warp in the inner regions of the disc. With the possible presence of a warped inner disc in MWC 758 projecting shadows in the outer regions, this possibility has not to be discarded. Indeed, the two other disks known to feature an inner warped disk, HD 135344B and HD 142527, also have a large cavity and spirals (Casassus et al. 2015a; Stolker et al. 2016; Christiaens et al. 2014). It is also still unclear if a planet inside the cavity, with a non-coplanar or eccentric orbit, might produce such spirals.

Finally, if massive enough, dust clumps might interact with, or even produce, spirals (Baruteau \& Zhu 2016; van der Marel et al. 2016b). The spiral propagating outwards in the north direction crosses, and maybe starts from, the south dust clump. We also observe that the pitch angle of this spiral increases in the vicinity of the north dust clump, both in near-IR and in the dust emission. However, this scenario is not able to produce the southeast spiral which is not cospatial with any of the two dust clumps. It would also be surprising that the south clump is able to produce a large spiral, while the north dust clump, about 3 times more massive, is not. 


\section{Conclusion}

The transitional disk around the Herbig star MWC 758 was previously known to feature a large cavity empty of millimeter grains, two large spirals observed in IR polarized emission and at least one asymmetry in the dust distribution. We present new ALMA observations at an angular resolution of about 0 ." 1-0." 2, which comprises dust emission at $\sim 0.88$ millimeter, and the ${ }^{13} \mathrm{CO}$ and $\mathrm{C}^{18} \mathrm{O} J=3-2$ emission lines. Our main findings about this system are:

1. The outer disk features two large dust clumps, localized at 47 and 82 au for the south and north clumps, respectively. The local increase in the dust surface density is of $\sim 2.5$ for the south clump and of $\sim 6.5$ for the north clump compared with values encountered at other azimuthal angles. The two dust concentrations are associated with elongated structures which are tracing a double-ring structure.

2. The main hypothesis for such dust concentrations is the dust trapping scenario, where a local gas pressure/density maximum aerodynamically accretes millimeter-size grains. The modeling of the gas and dust emission reveals smoother radial variations in the gas densities than in the dust. We also did not find any specific gas concentration cospatial with the dust clumps. This suggests lower local variations in the gas surface density, probably by no more than $20 \%-30 \%$. Additionally, the dust clumps appear to be in eccentric positions compared with the underlying doublering structure and the gas distribution, in regions with strong gradients in the surface density.

3. We observe the spirals, previously detected in near-IR scattered light, in the ${ }^{13} \mathrm{CO} J=3-2$ peak emission lines and in the continuum emission. The ${ }^{13} \mathrm{CO}$ peak emission is optically thick and mainly traces variations of the disk temperature while the dust emission is optically thin and probes the disk midplane. The spirals in IR and in the ${ }^{13} \mathrm{CO} J=3-2$ are located at the same radius while the spirals in continuum emission are located slightly at larger radius. This small offset in the radial location might be due to the spirals width and/or due to the vertical propagation of the spirals, which might curl toward the central star at the disk surface.

4. Two arc-like features, designated 3 and 4 in Figure 11, were previously observed in near-infrared. Our new ALMA observations show that these structures correspond to emission scattered at the inner edge of the two dust clumps.

5. Dust emission at millimeter wavelengths is detected at the position of the central star and reveals the presence of a small inner disk. The detection in the cavity of a twist in the velocity curves, as well as indications of a shadow projected toward the west outer region, suggest that this disk is mildly warped.

6. Our observations are consistent with the existence of two massive planets. A planet in the inner region of the disk which carves the cavity, and another planet in the outer regions to produce the spirals and the dark ring between the two dust clumps. In this scenario, the dust vortices at the origin of the dust clumps are probably triggered by the RWI due to the large radial gradients in the gas surface density. However, other possibilities exist and we discuss also other processes that might be able to produce the features encountered in the disk.

We acknowledge the anonymous referee for constructive comments and useful suggestions that improved this paper. Y.B. and A.I. acknowledge support from the NASA Origins of Solar Systems program through award NNX15AB06G. A.I. acknowledges support from the NSF Grant No. AST-1535809. E.W. acknowledges support from the NRAO Student Observing Support Grant No. AST-0836064. J.M.C. acknowledges support from the National Aeronautics and Space Administration under grant No. 15XRP15 20140 issued through the Exoplanets Research Program. Y-W.T. is supported by the Ministry of Science and Technology (MoST) in Taiwan through grant MoST 103-2119-M-001-010-MY2. This paper makes use of the following ALMA data ADS/JAO. ALMA2012.1.00725.S. ALMA is a partnership of ESO (representing its member states), NSF (USA) and NINS (Japan), together with NRC (Canada) and NSC and ASIAA (Taiwan) and KASI (Republic of Korea), in cooperation with the Republic of Chile. The Joint ALMA Observatory is operated by ESO, AUI/NRAO and NAOJ. The National Radio Astronomy Observatory is a facility of the National Science Foundation operated under cooperative agreement by Associated Universities, Inc. This work has also made use of data from the European Space Agency (ESA) mission Gaia (http:// www.cosmos.esa.int/gaia), processed by the Gaia Data Processing and Analysis Consortium (DPAC, http://www. cosmos.esa.int/web/gaia/dpac/consortium). Funding for the DPAC has been provided by national institutions, in particular the institutions participating in the Gaia Multilateral Agreement. The description of the Gaia mission is described in Gaia Collaboration et al. (2016b) and the data release 1 in Gaia Collaboration et al. (2016a).

\section{ORCID iDs}

Y. Boehler (1) https://orcid.org/0000-0002-8692-8744 A. Isella (i) https://orcid.org/0000-0001-8061-2207 M. Benisty (1) https://orcid.org/0000-0002-7695-7605 J. Carpenter (i) https://orcid.org/0000-0003-2251-0602 Bo-Ting Shen (ㄴ) https://orcid.org/0000-0003-3628-9187 Ya-Wen Tang (1) https://orcid.org/0000-0002-0675-276X L. Perez 느 https://orcid.org/0000-0002-1199-9564

\section{References}

ALMA Partnership, Brogan, C. L., Pérez, L. M., et al. 2015, ApJL, 808, L3 Anders, E., \& Grevesse, N. 1989, GeCoA, 53, 197

Andrews, S. M., Wilner, D. J., Espaillat, C., et al. 2011, ApJ, 732, 42 Bae, J., Zhu, Z., \& Hartmann, L. 2017, arXiv:1706.03066

Baruteau, C., Crida, A., Paardekooper, S.-J., et al. 2014, Protostars and Planets VI, 667

Baruteau, C., \& Zhu, Z. 2016, MNRAS, 458, 3927

Benisty, M., Juhasz, A., Boccaletti, A., et al. 2015, A\&A, 578, L6

Boehler, Y., Weaver, E., Isella, A., et al. 2017, ApJ, 840, 60

Casassus, S., Marino, S., Pérez, S., et al. 2015a, ApJ, 811, 92

Casassus, S., Wright, C. M., Marino, S., et al. 2015b, ApJ, 812, 126

Christiaens, V., Casassus, S., Perez, S., van der Plas, G., \& Ménard, F. 2014 ApJL, 785, L12

Dipierro, G., Laibe, G., Price, D. J., \& Lodato, G. 2016, MNRAS, 459, L1

Dong, R., van der Marel, N., Hashimoto, J., et al. 2017, ApJ, 836, 201

Dong, R., Zhu, Z., Rafikov, R. R., \& Stone, J. M. 2015, ApJL, 809, L5

Dullemond, C. P., Juhasz, A., Pohl, A., et al. 2012, Astrophysics Source Code Library, ascl:1202.015

Eisner, J. A., Lane, B. F., Hillenbrand, L. A., Akeson, R. L., \& Sargent, A. I. 2004, ApJ, 613, 1049 
Facchini, S., Juhász, A., \& Lodato, G. 2018, MNRAS, 473, 4459

Fedele, D., Carney, M., Hogerheijde, M. R., et al. 2017, A\&A, 600, A72

Gaia Collaboration, Brown, A. G. A., Vallenari, A., et al. 2016a, A\&A, 595, A2

Gaia Collaboration, Prusti, T., de Bruijne, J. H. J., et al. 2016b, A\&A, 595, A1

Gonzalez, J.-F., Laibe, G., Maddison, S. T., Pinte, C., \& Ménard, F. 2015, MNRAS, 454, L36

Grady, C. A., Muto, T., Hashimoto, J., et al. 2013, ApJ, 762, 48

Guilera, O. M., \& Sándor, Z. 2017, A\&A, 604, A10

Isella, A., Guidi, G., Testi, L., et al. 2016, PhRvL, 117, 25

Isella, A., Natta, A., Wilner, D., Carpenter, J. M., \& Testi, L. 2010, ApJ, 725,1735

Isella, A., Tatulli, E., Natta, A., \& Testi, L. 2008, A\&A, 483, L13

Johansen, A., Youdin, A., \& Klahr, H. 2009, ApJ, 697, 1269

Kley, W., \& Nelson, R. P. 2012, ARA\&A, 50, 211

Kratter, K., \& Lodato, G. 2016, ARA\&A, 54, 271

Kraus, S., Kreplin, A., Fukugawa, M., et al. 2017, ApJL, 848, L11

Lesur, G., \& Papaloizou, J. C. B. 2010, A\&A, 513, A60

Lobo Gomes, A., Klahr, H., Uribe, A. L., Pinilla, P., \& Surville, C. 2015, ApJ, 810,94

Marino, S., Casassus, S., Perez, S., et al. 2015b, ApJ, 813, 76

Marino, S., Perez, S., \& Casassus, S. 2015a, ApJL, 798, L44

McMullin, J. P., Waters, B., Schiebel, D., Young, W., \& Golap, K. 2007, adass XVI, 376, 127

Montesinos, M., Perez, S., Casassus, S., et al. 2016, ApJL, 823, L8

Muto, T., Tsukagoshi, T., Momose, M., et al. 2015, PASJ, 67, 122

Nelson, R. P., Gressel, O., \& Umurhan, O. M. 2013, MNRAS, 435, 2610
Owen, J. E., Ercolano, B., \& Clarke, C. J. 2011, MNRAS, 412, 13

Pérez, L. M., Carpenter, J. M., Andrews, S. M., et al. 2016, Sci, 353, 1519

Pérez, L. M., Isella, A., Carpenter, J. M., \& Chandler, C. J. 2014, ApJL, 783, L13

Pinilla, P., Birnstiel, T., Ricci, L., et al. 2012, A\&A, 538, A114

Pinilla, P., de Juan Ovelar, M., Ataiee, S., et al. 2015, A\&A, 573, A9

Qi, C., D'Alessio, P., Öberg, K. I., et al. 2011, ApJ, 740, 84

Quillen, A. C., Varnière, P., Minchev, I., \& Frank, A. 2005, AJ, 129, 2481

Ragusa, E., Dipierro, G., Lodato, G., Laibe, G., \& Price, D. J. 2017, MNRAS, 464, 1449

Regály, Z., Juhász, A., Sándor, Z., \& Dullemond, C. P. 2012, MNRAS, 419, 1701

Reggiani, M., Christiaens, V., Absil, O., et al. 2017, arXiv:1710.11393

Ruge, J. P., Flock, M., Wolf, S., et al. 2016, A\&A, 590, A17

Stolker, T., Dominik, C., Avenhaus, H., et al. 2016, A\&A, 595, A113

Tang, Y.-W., Guilloteau, S., Dutrey, A., et al. 2017, ApJ, 840, 32

van den Ancker, M. E., de Winter, D., \& Tjin A Djie, H. R. E. 1998, A\&A, 330, 145

van der Marel, N., Cazzoletti, P., Pinilla, P., \& Garufi, A. 2016b, ApJ, 832, 178

van der Marel, N., van Dishoeck, E. F., Bruderer, S., et al. 2013, Sci, 340, 1199

van der Marel, N., van Dishoeck, E. F., Bruderer, S., et al. 2016a, A\&A, 585, A58

van der Marel, N., van Dishoeck, E. F., Bruderer, S., Perez, L., \& Isella, A. 2015, A\&A, 579, A106

van Leeuwen, F. 2007, A\&A, 474, 653

Zhang, K., Blake, G. A., \& Bergin, E. A. 2015, ApJL, 806, L7

Zhu, Z., Dong, R., Stone, J. M., \& Rafikov, R. R. 2015, ApJ, 813, 88 